\title{
No association of serum sTREM2 and MRI-based brain volume in elderly people living in a rural community: a 7-year follow-up study
}

\section{Ryuzo Orihashi}

Saga University Faculty of Medicine: Saga Daigaku Igakubu

Yoshito Mizoguchi (D ymizo@cc.saga-u.ac.jp )

Saga University

\section{Yoshiomi Imamura}

Saga University Faculty of Medicine: Saga Daigaku Igakubu

\section{Shigeto Yamada}

Saga University Faculty of Medicine: Saga Daigaku Igakubu

\section{Akira Monji}

Saga University Hospital: Saga Daigaku Igakubu Fuzoku Byoin

\section{Research}

Keywords: sTREM2, cognitive function, MRI, voxel-based morphometry

Posted Date: December 16th, 2020

DOI: https://doi.org/10.21203/rs.3.rs-127416/v1

License: (9) This work is licensed under a Creative Commons Attribution 4.0 International License. Read Full License 


\section{Abstract}

\section{Background}

This study aimed to investigate the association between serum levels of soluble triggering receptor expressed on myeloid cells 2 (sTREM2), a soluble form of an innate immune receptor expressed on the microglia, and brain volume in elderly people.

\section{Methods}

The survey was conducted twice in Kurokawa-cho, Imari, Saga Prefecture, Japan, among people aged 65 years and older. We collected data from 596 residents. Serum sTREM2 level measurements, brain MRI, Mini-Mental State Examination (MMSE), and Clinical Dementia Rating (CDR) were performed at Time 1 (2009-2011). Follow-up brain MRI, MMSE, and CDR were performed at Time 2 (2016-2017). The interval between Time 1 and Time 2 was approximately 7 years. Sixty-nine participants ( 16 men, mean age 72.69 \pm 3.18 years; 53 women, mean age $72.68 \pm 4.64$ years) completed this study. We analyzed the correlation between serum sTREM2 levels (Time 1) and brain volume (Time 1, Time 2, and Time 1-Time 2 difference) using voxel-based morphometry implemented with Statistical Parametric Mapping.

Results

Participants in this study had lower MMSE and CDR scores 7 years after the baseline evaluation. However, analyses at the cluster level by applying multiple comparison corrections (family wise error; $\mathrm{P}<$ 0.05 ) showed no correlation between serum sTREM2 levels and volume of different brain regions, either cross-sectional or longitudinal.

Conclusions

Serum STREM2 level could not serve as an immune biomarker of aging-related volume changes in brain regions closely related to cognitive function in elderly people aged 65 years and above.

\section{Full Text}

This preprint is available for download as a PDF.

\section{Figures}




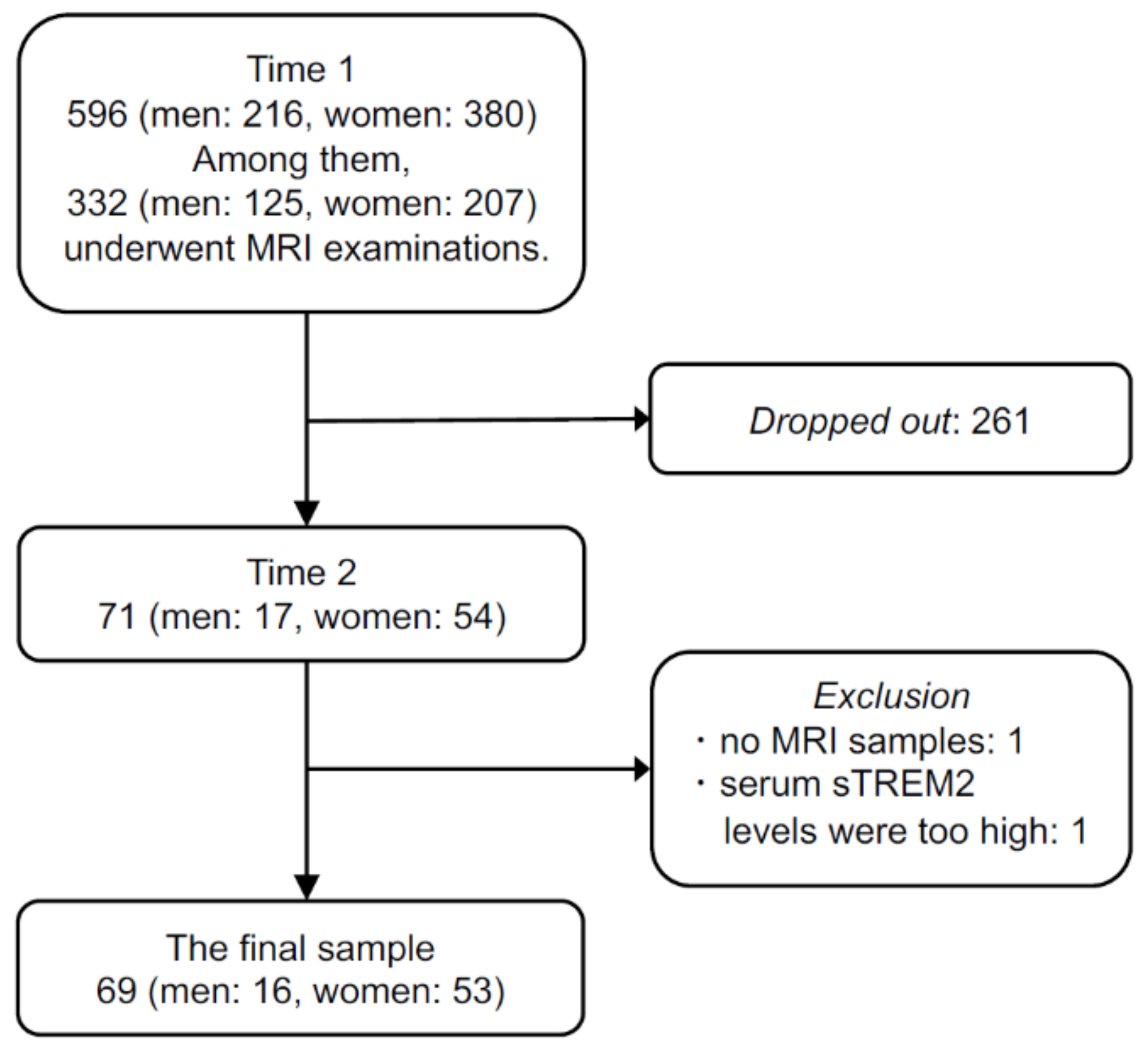

Figure 1

Flow chart depicting selection procedure. 

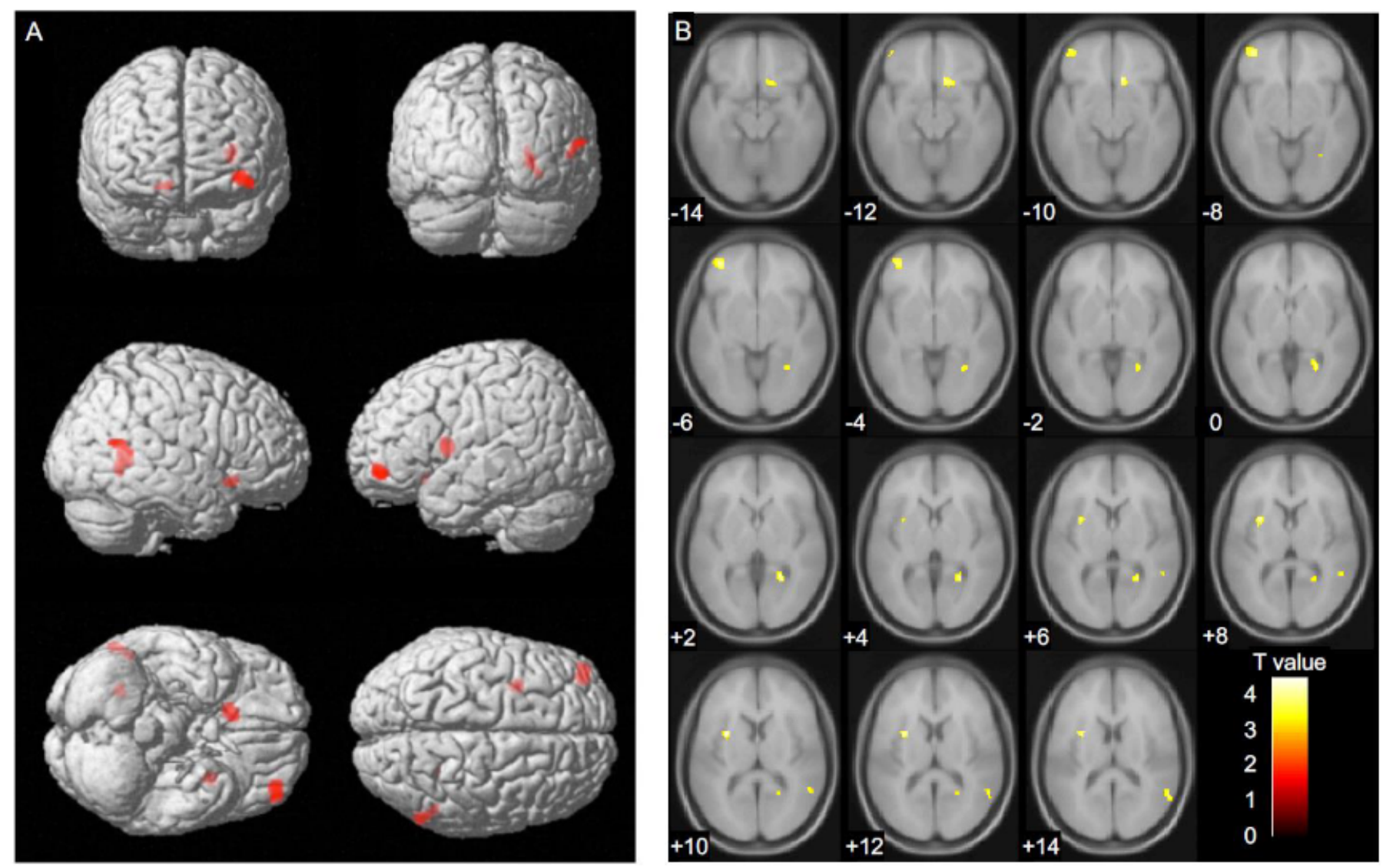

Figure 2

Voxel-based morphometry findings: Association between serum sTREM2 levels (Time 1) and brain volume (Time 2). Positive correlation between serum sTREM2 levels (Time 1) and brain volume (Time 2), as shown by multiple regression analysis. Volumes of the important regions are shown in $(A)$ whole brain images and (B) axial images. The T value applies to (B) axial images. 

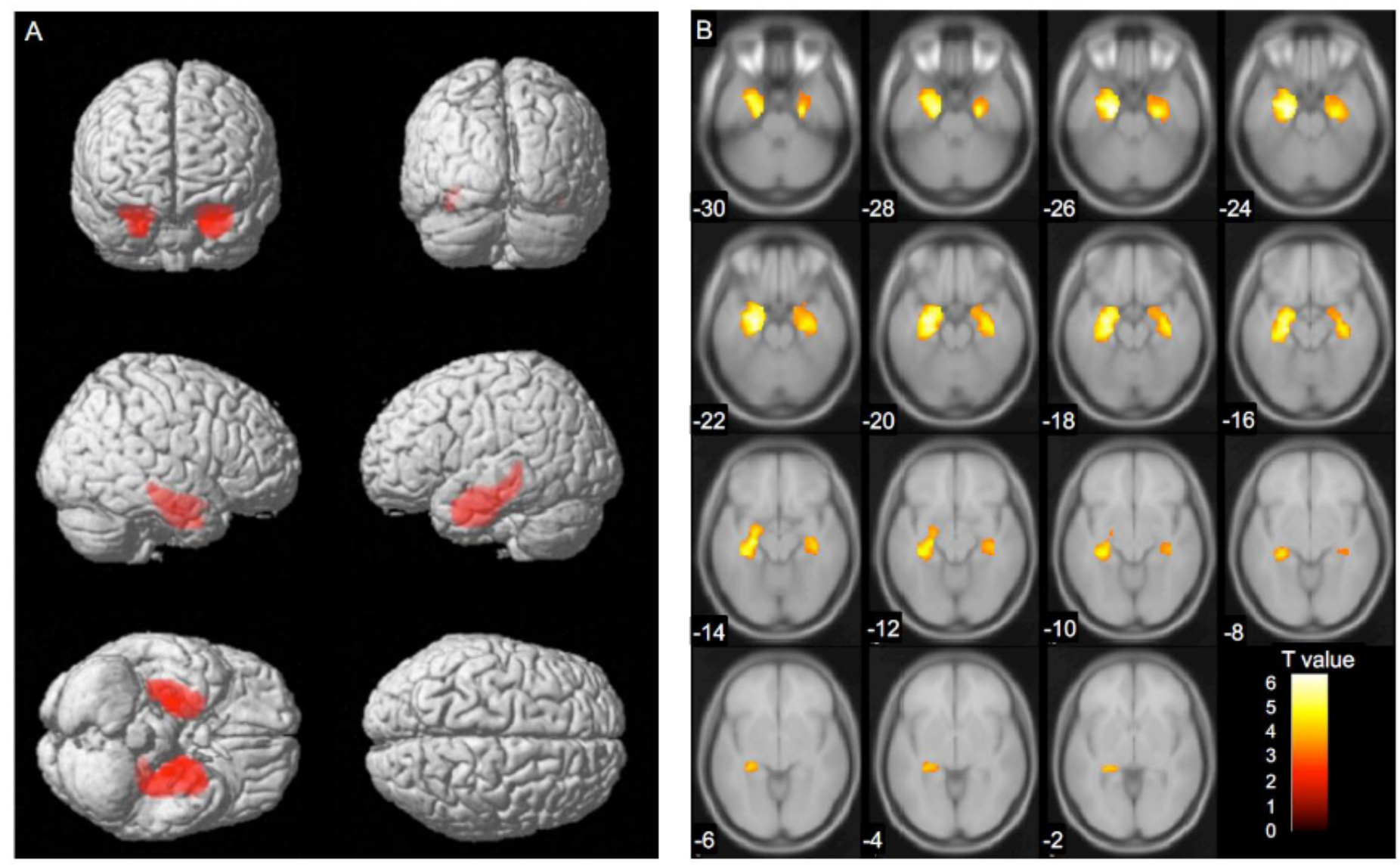

\section{Figure 3}

Voxel-based morphometry findings: Association between changes in CDR scores and brain volume (Time 2). Association between lower CDR scores and decreased brain volume (Time 2) evaluated using multiple regression analysis. Significant clusters containing the left and the right hippocampus are shown in (A) whole brain images and (B) axial images. The $T$ value applies to (B) axial images. 